Disclosure(s) Nothing to disclose

\section{O29 PHARMACOMETRIC MODELLING OF FREE THYROXINE DYNAMICS AFTER INITIATION OF ANTITHYROID DRUG TREATMENT IN CHILDREN WITH GRAVES' DISEASE: A TOOL FOR PERSONALIZED DRUG DOSING IN CHILDREN}

${ }^{1} \mathrm{G}$ Koch*, ${ }^{2} \mathrm{~V}$ Gotta, ${ }^{2} \mathrm{C}$ Ollagnier, ${ }^{3} \mathrm{D}$ Konrad, ${ }^{4} \mathrm{C}$ Flück, ${ }^{5} \mathrm{D}$ L'Allemand, ${ }^{6} \mathrm{M}$ Steigert, ${ }^{7} \mathrm{~A}$ Rohner, ${ }^{8} \mathrm{~F}$ Bachmann, ${ }^{8}$ J Schropp, ${ }^{7} \mathrm{G}$ Szinnai, ${ }^{7} \mathrm{M}$ Pfister. ${ }^{1}$ Pediatric Pharmacology and Pharmacometrics, University Children's Hospital Basel UKBB; ${ }^{2}$ University Children's Hospital Basel UKBB, Basel; ${ }^{3}$ University Children's Hospital Zürich, Zürich; ${ }^{4}$ University Hospital Bern, Bern; ${ }^{5}$ Ostschweizer Kinderspital, St. Gallen; ${ }^{6}$ Kantonspital Graubünden, Chur; ${ }^{7}$ University Children's Hospital Basel (UKBB), Basel, Switzerland; ${ }^{8}$ University of Konstanz, Konstanz, Germany

\subsection{6/archdischild-2019-esdppp.29}

Background Thyroid hormones are essential for normal growth and puberty in children and adolescents. Graves' disease (GD) is a rare autoimmune disorder associated with thyroid hormone over-production and occurs in 0.5-2:100'000 children (mostly females) in Europe. In contrast to adults, first line treatment of GD is long-term antithyroid drug (ATD) therapy, and radioiodine ablation is usually being reserved for patients after puberty. However, treatment of acquired hyperthyroidism with ATD is difficult since 1) paediatric patients differ considerable in age, weight and diseases severity and 2) minimal ATD dose should be used to avoid mild side effects (occurring in $6-35 \%$ of patients), and rare severe side effects such as agranulocytosis and liver failure.

Methods Longitudinal clinical and laboratory data from a Swiss multicentre cohort were analysed retrospectively. Nonlinear mixed effects modelling was applied to characterize dynamics of free thyroxine (FT4) during the first 3 months of treatment with carbimazole. Sex, co-medications such as propranolol, and thyroid stimulating hormone receptor antibody were tested as covariates on model parameters. Reference range for FT4 was derived from target levels in clinical practice $(12-22 \mathrm{pmol} / \mathrm{L})$.

Results Study cohort comprised 45 children with GD, 78\% females, median age 12.2 years $(\mathrm{IQR}=[8.7,13.6]))$ with a total of 181 visits. FT4 baseline at diagnosis of GD was 62.3 $\mathrm{pmol} / \mathrm{L}(\mathrm{IQR}=[45.7,86.7])$. None of the tested factors showed significant covariate effects. The model allows individual simulations of optimal ATD dosing strategies (starting and maintenance dose) for different GD severities, ages and weights at start of therapy. Personalized dosing examples will be presented.

Conclusions Developed pharmacometric model is able to predict dynamics of FT4 in children with GD depending on four parameters: ATD dose $/ \mathrm{kg} / \mathrm{d}$, age, weight and disease severity, and can be applied to personalize dosing regimen to avoid over- or under dosing.

Disclosure(s) Nothing to disclose

\section{O30 A COMPREHENSIVE ANALYSIS OF ONTOGENY OF RENAL DRUG TRANSPORTERS: MRNA ANALYSES, QUANTITATIVE PROTEOMICS AND LOCALIZATION}

${ }^{1,2,3}$ KWK Cheung*, ${ }^{4} \mathrm{BD}$ van Groen*, ${ }^{4,5} \mathrm{E}$ Spaans, ${ }^{6} \mathrm{MD}$ van Borselen, ${ }^{7} \mathrm{ACJM}$ de Bruijn, ${ }^{7} Y$ Simons-Oosterhuis, ${ }^{4} \mathrm{D}$ Tibboel, ${ }^{7} \mathrm{JN}$ Samsom, ${ }^{8} \mathrm{RM}$ Verdijk, ${ }^{9} \mathrm{~B}$ Smeets, ${ }^{10} \mathrm{~L}$ Zhang, ${ }^{2} \mathrm{~S}$-M Huang, ${ }^{1} \mathrm{KM}$ Giacomini ${ }^{* *},{ }^{4,6} \mathrm{SN}$ de Wildt**. ${ }^{*}$ University of California, San Francisco, San Francisco, CA; ${ }^{2}$ Office of Clinical Pharmacology, Office of Translational Sciences, Center for Drug Evaluation and Research, U.S. Food and Drug Administration, Silver Spring, MD; ${ }^{3}$ Oak Ridge Institute for Science and Education (ORISE Fellow), Oak Ridge, TN, USA; ${ }^{4}$ Intensive Care and Department of Pediatric Surgery, Erasmus MC-Sophia Children's Hospital, Rotterdam; ${ }^{5}$ CDTS Consulting BV and SDD Consulting BV, Etten-Leur; ${ }^{6}$ Department of Pharmacology and Toxicology, Radboud University, Nijmegen; ${ }^{7}$ Department of Pediatrics, Erasmus MC-Sophia Children's Hospital; ${ }^{8}$ Department of Pathology, Erasmus MC, Rotterdam; ${ }^{9}$ Department of Pathology, Radboudumc, Nijmegen, The Netherlands; ${ }^{10}$ Office of Research and Standards, Office of Generic Drugs, Center for Drug Evaluation and Research, U.S. Food and Drug Administration, Silver Spring, MD, USA

\subsection{6/archdischild-2019-esdppp.30}

Background Postnatal developmental changes of human renal membrane transporters, which are key players of disposition of renally cleared drugs and endogenous substrates, are largely unknown. This study aimed to characterize the ontogeny of 11 human renal transporters to understand changes in the renal clearance of substrate drugs in children.

Methods mRNA levels of known renal transporters: BCRP, MATE1, MATE2-K, MDR1, MRP2, MRP4, URAT1, GLUT2, OAT1, OAT3 and OCT2, and the transcription factor PXR were measured with RT-qPCR in 184 human postmortem frozen renal cortical tissues (preterm newborns - adults; 1 day-75 yrs old) from individuals of European and African descent. Protein expression of all but MRP2, MRP4 and PXR was quantified with LC-MS/MS SRM in 62 of those samples (term newborns - adults; 1 day-29 yrs old). Localization of MRP4 was tested with immunohistochemistry.

Results Expression levels of MDR1, URAT1, OAT1, OAT3, and OCT2 increased with age, but levels of MATE1 and GLUT2 were stable from birth. Protein levels of MATE2-K and BCRP showed no difference from newborns to adults despite age-related changes in mRNA expression. MRP2, MRP4 and PXR expression levels were stable. MRP4 localization in pediatric samples was similar to that in adult samples. Conclusion Renal drug transporters exhibited different rates and patterns of maturation, suggesting that renal handling of both endogenous and exogenous compounds may change with age. It is important to consider ontogeny of renal transporters during pediatric drug development.

Disclosure(s) The authors declare that the research was conducted in the absence of any commercial or financial relationships that could be construed as a potential conflict of interest. Views expressed in this paper are those of authors and do not necessarily reflect the official views or policies of the FDA; nor does any mention of trade names, commercial practices, or organization imply endorsement by the U.S. Government.

*Contributed equally,

**Contributed equally 